\title{
DETECTION AND SEGMENTATION OF ENDOSCOPIC ARTEFACTS AND DISEASES USING DEEP ARCHITECTURES
}

\author{
Nhan T. Nguyen*, Dat Q. Tran*, Dung B. Nguyen \\ Medical Imaging Department, Vingroup Big Data Institute (VinBDI), Hanoi, Vietnam \\ $\{\mathrm{v} \cdot \mathrm{nhannt} 64 ; \mathrm{v} \cdot$ dattq13; v.dungnb1\}@vinbdi.org
}

\begin{abstract}
We describe in this paper our deep learning-based approach for the EndoCV2020 challenge, which aims to detect and segment either artefacts or diseases in endoscopic images. For the detection task, we propose to train and optimize EfficientDet—a state-of-the-art detector-with different EfficientNet backbones using Focal loss. By ensembling multiple detectors, we obtain a mean average precision (mAP) of 0.2524 on EDD2020 and 0.2202 on EAD2020. For the segmentation task, two different architectures are proposed: UNet with EfficientNet-B3 encoder and Feature Pyramid Network (FPN) with dilated ResNet-50 encoder. Each of them is trained with an auxiliary classification branch. Our model ensemble reports an sscore of 0.5972 on EAD2020 and 0.701 on EDD2020, which were among the top submitters of both challenges.
\end{abstract}

\section{INTRODUCTION}

Disease detection and segmentation in endoscopic imaging play an important role in the early detection of numerous cancers, such as gastric, colorectal, and bladder cancers [1]. Meanwhile, the detection and segmentation of endoscopic artefacts is necessary for image reconstruction and quality assertion [2]. Many approaches [3, 4, 5] have been proposed to detect and segment artefacts and diseases in endoscopy. This paper describes our solution for the EndoCV2020 challenge, which consists of two tracks: one deals with artefacts (EAD2020) and the other one is for diseases (EDD2020). Each track is divided into two tasks: detection and segmentation. We tackle both tasks in both tracks by exploiting state-of-the-art deep architectures like EfficientDet [6] and U-Net [7] with variants of EfficientNet [8] and ResNet [9] as backbones. In the next sections, we provide a short description of the datasets, the details of the proposed approach, and experimental results.

\footnotetext{
* Equal contribution.
}

\section{DATASETS}

EDD2020 [1] is a comprehensive dataset established to benchmark algorithms for disease detection and segmentation in endoscopy. It is annotated for 5 different disease classes, including BE, Suspicious, HGD, Cancer, and Polyp. The dataset comes with bounding boxes for disease detection and with masked image annotations for semantic segmentation. The training set includes total 386 endoscopy frames, each of which is annotated with either single or multiple diseases. Regions of the same class are merged into a single mask, while a bounding box of multiple classes is treated as separate boxes with the same location. Figure 1 shows the number of bounding boxes for each disease class.

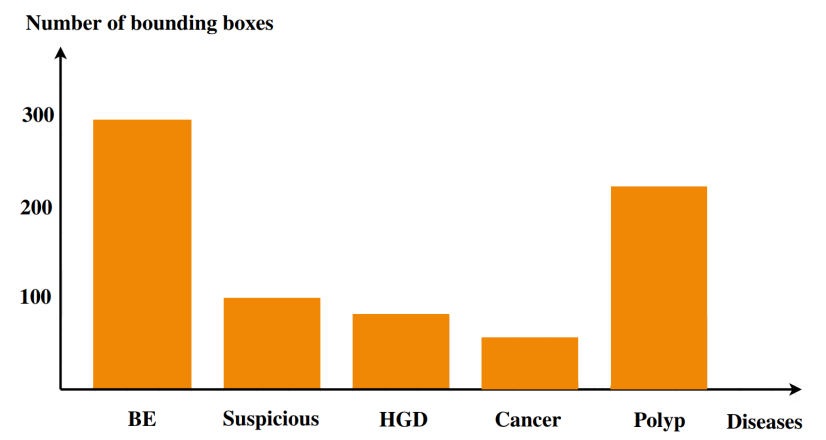

Fig. 1. The number of bounding boxes for each disease class in training set provided by the EDD2020 dataset.

EAD2020 [10], on the other hand, is used for the track of endoscopy artefact detection and segmentation. The training set contains 2,531 annotated frames for 8 artefact classes, including specularity, bubbles, saturation, contrast, blood, instrument, blur, and imaging artefacts. Note that only first 5 classes are used for the segmentation task.

\section{PROPOSED METHODS}

\subsection{Multi-class detection task}

Detection network: For the detection task, we deployed EfficientDet [6], currently a state-of-the-art architecture for 
medRxiv preprint doi: https://doi.org/10.1101/2020.04.17.20070201; this version posted April 22, 2020. The copyright holder for this preprint (which was not certified by peer review) is the author/funder, who has granted medRxiv a license to display the preprint in perpetuity. It is made available under a CC-BY-NC-ND 4.0 International license .

object detection. It employs EfficientNet [8] as the backbone network, BiFPN as the feature network, and shared class/box prediction network. Both BiFPN layers and class/box net layers are repeated multiple times based on different resource constraints. Figure 2 illustrates the EfficientDet architecture.

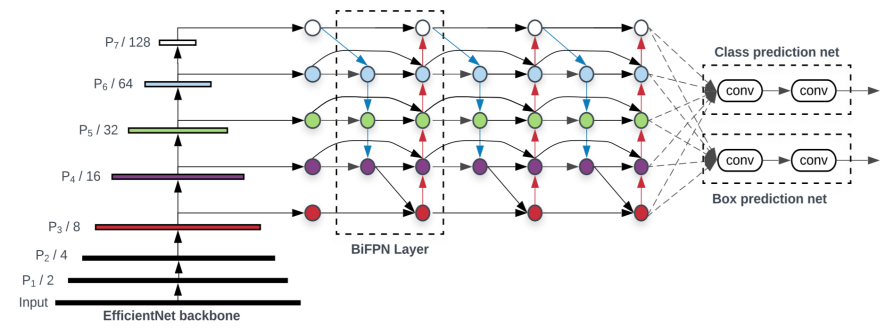

Fig. 2. The EfficientDet architecture. The class prediction network was modified for providing the probabilities of 5 disease classes. The figure was reproduced from Tan et al. [6].

Training procedure: Due to the limited training data available (386 images in EDD2020 and 2531 images in EAD2020), we use various data augmentation techniques, including random shift, random crop, rotation, scale, horizontal flip, vertical flip, blur, Gauss noise, sharpen, emboss, and contrast. In particular, we found that the use of mixup could significantly reduce the overfitting. Given $x_{1}$ and $x_{2}$ as input images, the mixup image $\tilde{x}$ is constructed as
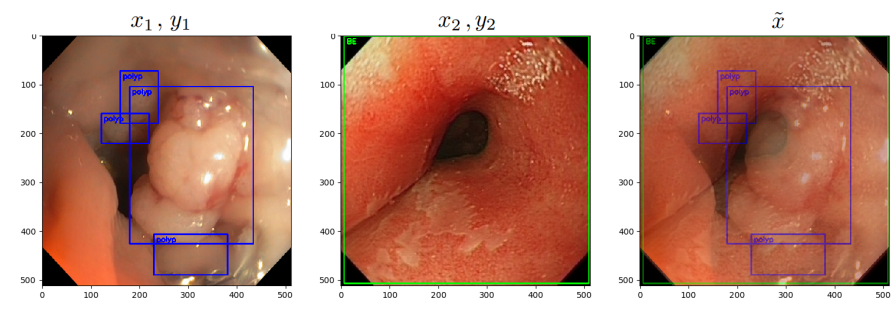

Fig. 3. Mixup visualization with $\lambda=0.5$.

$$
\begin{gathered}
\tilde{x}=\lambda x_{1}+(1-\lambda) x_{2}, \\
\tilde{x} \stackrel{\text { Network }}{\longrightarrow} \hat{y} .
\end{gathered}
$$

During training, our goal is to minimize the MixLoss $\mathcal{L}_{\text {mixup }}$, which is expressed as

$$
\mathcal{L}_{\text {mixup }}=\lambda \mathcal{L}\left(\hat{y}, y_{1}\right)+(1-\lambda) \mathcal{L}\left(\hat{y}, y_{2}\right) .
$$

where the symbol $\mathcal{L}$ denotes the Focal loss [11] and $\lambda$ is drawn from $\beta(0.75,0.75)$ distribution; $y_{1}$ and $y_{2}$ are the groundtruth labels, while $\hat{y}$ is the predicted label produced by the network. Fig. 3 visualizes a mixup example with $\lambda$ being fixed to 0.5 .

Our detectors are optimized by the gradient decent using Adam update rule [12] with weight decay. In addition, cyclical learning rate [13] with restarts is also used. The ensemble of 6 models with different backbones (D0, D1, D2, D3, D4, and D5) using weighted box fusion [14] serves as our final model. Additionally, we search for the non-maximum suppression (NMS) threshold and the confidence threshold for different categories so that the resulting score $(0.5 \times \mathrm{mAP}+$ $0.5 \times$ IOU) is maximized.

\subsection{Multi-class segmentation task}

Segmentation network: We propose two different architectures for this task: U-Net with EfficientNet encoders and BiFPN with ResNet encoders.

U-Net: Our first network design makes use of U-Net with EfficientNetB3/B4 as backbones. We keep the original strides between blocks in EfficientNet and extract the feature maps from the last 5 blocks for the segmentation. A classification branch is used to provide the label predictions. The overall framework is depicted in Figure 4.

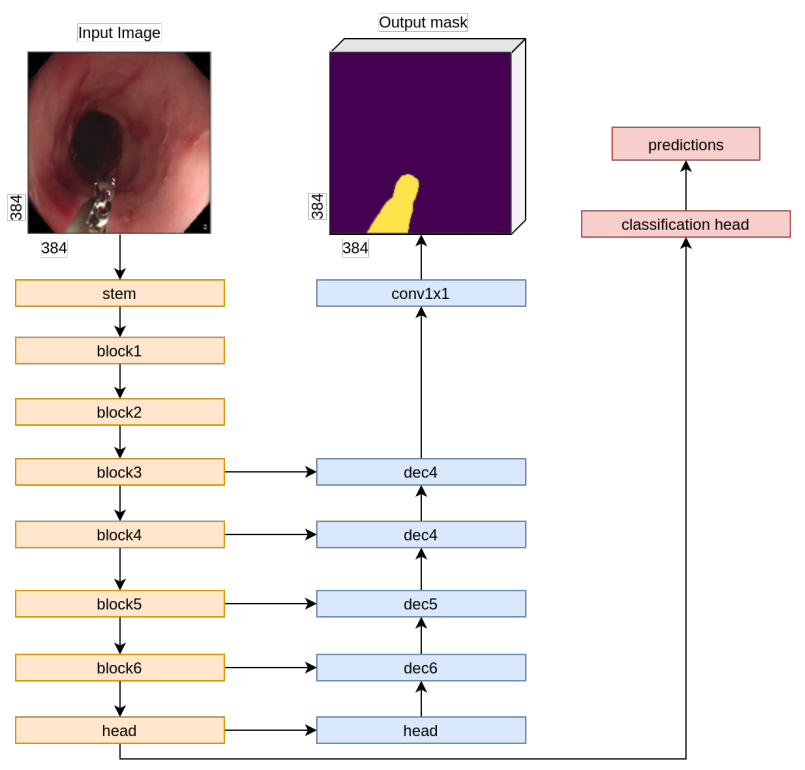

Fig. 4. The U-Net with EfficientNetB3/B4 encoder and a classification branch architecture.

BiFPN: To generate the segmentation output from the BiFPN features, we combine all levels of the BiFPN pyramid by following the design illustrated in Figure 5. Starting with the deepest BiFPN level (stride-32 output), we apply three upsampling stages to obtain the feature map of the stride- 4 output. An upsampling stage consists of a $3 \times 3$ Convolution, BatchNorm, ReLU and a $2 \times 2$ bilinear upsampling. This strategy is repeated for other BiFPN levels with strides of 16 , 8 , and 4 . The result is a set of feature maps at the same scale, which are then channel-wise concatenated. Finally, a $1 \times 1$ Convolution, $4 \times 4$ bilinear upsampling and Sigmoid activation are used to generate the mask at the image resolution. 


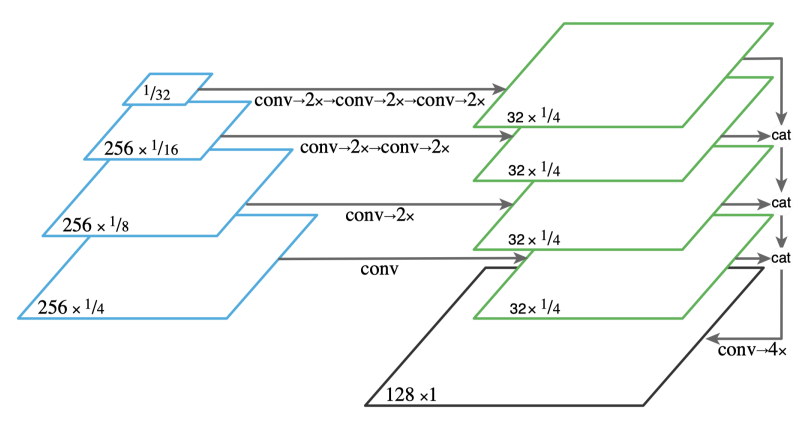

Fig. 5. The BiFPN decoder for semantic segmentation.

\begin{tabular}{c|c|c|c|c} 
Challenge & dscore & dstd & sscore & sstd \\
\hline EAD2020 & 0.2202 & 0.1029 & 0.5972 & 0.2765 \\
\hline EDD2020 & 0.2524 & 0.0948 & 0.7008 & 0.3211
\end{tabular}

Table 1. Detection and segmentation scores on the EndoCV2020 test set.

Training procedure: All models are trained end-to-end with additional supervision from the multi-label classification task. The image labels are obtained directly from the segmentation masks. For example, if an image has B.E. mask annotation then the B.E. label is 1. Due to class imbalance in the training dataset, we use Focal loss for the classification task. Our final loss is $\mathcal{L}=\mathcal{L}_{\text {seg }}+\lambda \times \mathcal{L}_{\text {cls }}$ where $\lambda=0.4$.

Inference: Relying solely on segmentation branch to predict masks will result in high false positives. Hence, we make use of the class predictions to remove masks. We search optimal classification thresholds to maximize the macro F1 score on the validation set. For every image, if the class probability is less than the optimal threshold then its predicted mask is completely removed.

\section{EXPERIMENTAL RESULTS}

Table 1 summarizes the detection and segmentation results of our submissions for both challenges. We describe the results of each sub-task below.

Results on the validation set of EDD2020 for the detection task are detailed in Table 2. Our best single model (i.e. EfficientDet-D5) obtained a detection score (dScore) of 0.41 . The best detection performance was provided by the ensemble model, which reported a dScore of 0.44 , a mean mAP of $0.36 \pm 0.05$, and an IoU of 0.52 . As shown in Table 1, our ensemble model yielded dScores of $0.2524 \pm 0.0948$ and $0.2202 \pm 0.1029$ on the hidden test sets of EDD2020 and EAD2020, respectively.

Results on validation sets for the segmentation task are provided in Table 3 and Table 4. On the EDD2020 validation set, our best single model achieved a Dice score of 0.854 and

\begin{tabular}{c|c|c|c} 
Method & dScore & mAP & IoU \\
\hline ED0 [6] & 0.23 & $0.13 \pm 0.04$ & 0.33 \\
\hline ED0, Augs & 0.34 & $0.26 \pm 0.07$ & 0.42 \\
\hline ED0, Augs, Mixup, CLR [15] & 0.40 & $0.30 \pm 0.05$ & 0.51 \\
\hline ED5, Augs, Mixup, CLR [15] & 0.41 & $0.29 \pm 0.05$ & 0.54 \\
\hline Ensemble (ED0-ED5), WBF [14] & 0.44 & $0.36 \pm 0.05$ & 0.52
\end{tabular}

Table 2. Experimental results on EDD2020 validation set.

an IoU of 0.832. On the EAD2020 validation set, we obtained a Dice score of 0.732 and an IoU of 0.578. As shown in Table 1 , our ensemble achieved a segmentation score (sscore) of 0.5972 in the EAD2020 challenge and an sscore of 0.7008 in the EDD2020 challenge, both of which were among the top results for the segmentation task of both tracks.

\begin{tabular}{c|c|c} 
Method & Dice & IoU \\
\hline UNet-EfficientNetB4 [8][7] & $0.8522 \pm 0.0221$ & $0.8279 \pm 0.0213$ \\
\hline BiFPN-ResNet50 & $0.8544 \pm 0.0232$ & $0.8317 \pm 0.0228$
\end{tabular}

Table 3. 5-fold cross-validation results on EDD2020.

\begin{tabular}{c|c|c} 
Method & Dice & IoU \\
\hline UNet-EfficientNetB4 & $0.7131 \pm 0.0379$ & $0.555 \pm 0.0451$ \\
\hline BiFPN-ResNet50 & $0.7325 \pm 0.0162$ & $0.578 \pm 0.0201$
\end{tabular}

Table 4. 3-fold cross-validation results on EAD2020.

\section{CONCLUSION}

We have described our solutions for the detection and segmentation tasks on both tracks of EndoCV2020: EAD for artefacts and EDD for diseases. By using EfficientDet for detection and U-Net/BiFPN for segmentation, we obtained significant results on both datasets, especially for the segmentation task. These results suggest that some of the deep architectures that are effective for natural images can also be useful for medical images like endoscopic ones, even with a small-size training datasets.

\section{REFERENCES}

[1] Dominique Lamarque Stefano Realdon Adam Bailey Renato Cannizzaro Noha Ghatwary Jens Rittscher Christian Daul James East Sharib Ali, Barbara Braden. Endoscopy disease detection and segmentation (edd2020), 2020.

[2] Sharib Ali, Felix Zhou, Christian Daul, Barbara Braden, Adam Bailey, Stefano Realdon, James East, Georges Wagnières, Victor Loschenov, Enrico Grisan, Walter Blondel, and Jens Rittscher. Endoscopy arti- 
medRxiv preprint doi: https://doi.org/10.1101/2020.04.17.20070201; this version posted April 22, 2020. The copyright holder for this preprint (which was not certified by peer review) is the author/funder, who has granted medRxiv a license to display the preprint in perpetuity.

It is made available under a CC-BY-NC-ND 4.0 International license .

fact detection (EAD 2019) challenge dataset. CoRR, abs/1905.03209, 2019.

[3] PS Hiremath, BV Dhandra, Iranna Humnabad, Ravindra Hegadi, and GG Rajput. Detection of esophageal cancer (necrosis) in the endoscopic images using color image segmentation. In Proceedings of second $\mathrm{Na}$ tional Conference on Document Analysis and Recognition (NCDAR-2003), Mandya, India, pages 417-422, 2003.

[4] Piotr Szczypiński, Artur Klepaczko, Marek Pazurek, and Piotr Daniel. Texture and color based image segmentation and pathology detection in capsule endoscopy videos. Computer methods and programs in biomedicine, 113(1):396-411, 2014.

[5] Eva Tuba, Milan Tuba, and Raka Jovanovic. An algorithm for automated segmentation for bleeding detection in endoscopic images. In 2017 International Joint Conference on Neural Networks (IJCNN), pages 45794586. IEEE, 2017.

[6] Mingxing Tan, Ruoming Pang, and Quoc V Le. Efficientdet: Scalable and efficient object detection. arXiv preprint arXiv:1911.09070, 2019.

[7] Olaf Ronneberger, Philipp Fischer, and Thomas Brox. U-net: Convolutional networks for biomedical image segmentation. arXiv preprint arXiv:1505.04597, 2015.

[8] Mingxing Tan and Quoc V Le. Efficientnet: Improving accuracy and efficiency through automl and model scaling. arXiv preprint arXiv:1905.11946, 2019.

[9] Kaiming He, Xiangyu Zhang, Shaoqing Ren, and Jian Sun. Deep residual learning for image recognition. In IEEE CVPR, pages 770-778, 2016.

[10] Sharib Ali, Felix Zhou, Adam Bailey, Barbara Braden, James East, Xin Lu, and Jens Rittscher. A deep learning framework for quality assessment and restoration in video endoscopy. CoRR, abs/1904.07073, 2019.

[11] Tsung-Yi Lin, Priya Goyal, Ross Girshick, Kaiming He, and Piotr Dollár. Focal loss for dense object detection. In Proceedings of the IEEE international conference on computer vision, pages 2980-2988, 2017.

[12] Diederik P Kingma and Jimmy Ba. Adam: A method for stochastic optimization. arXiv preprint arXiv:1412.6980, 2014.

[13] Leslie N Smith. Cyclical learning rates for training neural networks. In 2017 IEEE Winter Conference on Applications of Computer Vision (WACV), pages 464-472. IEEE, 2017.
[14] Roman Solovyev and Weimin Wang. Weighted boxes fusion: ensembling boxes for object detection models. arXiv preprint arXiv:1910.13302, 2019.

[15] Ilya Loshchilov and Frank Hutter. Decoupled weight decay regularization. arXiv preprint arXiv:1711.05101, 2017. 